Research Paper

\title{
Cellular glucose metabolism is essential for the reduction of cell-impermeable water-soluble tetrazolium (WST) dyes
}

\author{
Linna Xie ${ }^{1}$, Zichan Dai ${ }^{2}$, Chunxiu Pang ${ }^{1}$, Dexin Lin ${ }^{1,}$ and Min Zheng1,2, \\ 1. Department of Biochemistry and Molecular Biology, School of Basic Medical Sciences, Fujian Medical University, Fuzhou, Fujian, 350108, China. \\ 2. Fujian Key Laboratory for Translational Research in Cancer and Neurodegenerative Diseases, Institute for Translational Medicine, Fujian Medical \\ University, Fuzhou, Fujian, 350108, China. \\ $\triangle$ Corresponding authors: Min Zheng at mzheng@mail.fjmu.edu.cn and Dexin Lin at dexin9997@163.com. Tel. (+86) 591-22862007.
}

(c) Ivyspring International Publisher. This is an open access article distributed under the terms of the Creative Commons Attribution (CC BY-NC) license (https://creativecommons.org/licenses/by-nc/4.0/). See http://ivyspring.com/terms for full terms and conditions.

Received: 2018.02.20; Accepted: 2018.08.08; Published: 2018.09.07

\begin{abstract}
Water-soluble tetrazolium (WST) dyes, such as WST-1 and WST-8, are widely used in cell proliferation and anti-cell-growth drug screen assays. However, the underlying determinants for WST reduction are still largely unknown. In addition, application of tetrazolium-based assays to cellular glucose metabolism studies has not been fully explored. In the present study, we show here that WST-8 reduction is dependent on cellular glucose metabolism. In order to minimize the variance of live cell number during stimulation, we treated cells with different stimuli and performed tetrazolium-based assays within 6 hours. Withdrawal of medium glucose supply greatly attenuated WST-8 reduction but not intracellular ATP levels, while re-adding glucose reconstituted WST-8 reduction, indicating the effect was not due to cell death. The role of glucose on WST-8 reduction is specific since glutamine, fructose or galactose did not substitute for the effect of glucose on WST-8 reduction. Furthermore, inhibition of glucose transporters, intracellular glucose metabolic enzymes or EGFR-PI3K-Akt signaling also attenuated WST-8 reduction. In an attempt to screen inhibitors targeting cellular glucose metabolism from hyperglycemia-associated drugs, it turned out that HIV protease inhibitor, ritonavir, could largely block WST-8 reduction, but not cellular ATP level. Interestingly, ritonavir has been shown to acutely block glucose transport in vitro and in vivo. Taken together, our studies not only demonstrate an essential role of cellular glucose metabolism on WST-8 reduction, but also propose a novel application of tetrazolium-based assays in screening for inhibitors of cellular glucose metabolism when used in combination with ATP assay.
\end{abstract}

Key words: water-soluble tetrazolium, cellular glucose metabolism, glycolysis, EGFR-PI3K-Akt, ritonavir, drug screening.

\section{Introduction}

Tetrazolium salts are compounds containing a positively charged quaternary tetrazole ring core, which involves four nitrogen atoms surrounded by three aromatic groups [1]. The net charge on the tetrazolium salts determines whether these molecules are cell-permeable [2]. The cell-permeable MTT forms water insoluble needle-like formazan crystals [3]. By adding sulfonic, iodo, nitro and methoxy groups to the phenyl moieties, cell-impermeable tetrazolium salts, such as MTS, WST-1 and WST-8 (also known as Cell Counting Kit-8, CCK-8) were developed and form water-soluble formazan. This property enables WST to be utilized as a real-time assay for cellular metabolism, not just as an end-point assay. These compounds lose the ability to cross cellular membrane, and therefore require 1-methoxy-5methyl-phenazinium methyl sulfate (mPMS) as the electron carrier for trans-membrane electron transport [1].

Reduction of the central structure and breakdown of the tetrazole ring transforms the tetrazolium salts from weakly colored to brightly colored products, which are known as formazan [4]. This biochemical property underpins the use of these dyes in colorimetric assays [4]. Tetrazolium-based assays have been widely used as a powerful tool in microbiology, biochemistry and cell biology for more than half a century. Their use has increased 
exponentially since 1983 when Mosmann first introduced the use of tetrazolium salts in rapid colorimetric assays to determine cell proliferation [5]. Furthermore, its utilization in micro-plate makes it feasible for high-throughput drug screening [6].

Tetrazolium dye reduction is driven by intracellular reductants, such as NADH and $\mathrm{NAD}(\mathrm{P}) \mathrm{H}$ [7], which mainly come from glucose metabolism in cells cultured in-vitro. The correlation of cell-permeable tetrazolium MTT reduction and medium glucose supply was first investigated in 1991. A decrease of D-glucose concentration from the medium was accompanied by a decrease of MTT reduction in multiple human cancer cell lines [7]. Formazan production also increased as a function of the extracellular glucose concentration in both rat insulinoma INS-1 and islets cells [8]. However, the role of cellular glucose metabolism on cell-impermeable tetrazolium dye reduction has not been determined.

In this study, we investigated the role of cellular glucose metabolism on tetrazolium dye reduction. In order to minimize the variance of live cell number during stimulation, tetrazolium-based assays were performed within 6 hours post-stimulation. We demonstrated that withdrawal of extracellular glucose supply could greatly inhibit tetrazolium dye reduction, while re-addition of glucose could re-constitute tetrazolium dye reduction. Combined with an ATP assay, we demonstrated that the defect of WST-8 reduction was not a consequence of cell death. Inhibition of glucose transport and intracellular glucose metabolic enzymes could also greatly inhibit WST-8 reduction. In support of this, inhibition of EGFR-PI3K-Akt pathway, which had been shown to regulate cellular glucose metabolism $[9,10]$ also had an inhibitory effect on WST-8 reduction. In an attempt to investigate the effect of hyperglycemia-associated drugs on WST-8 reduction, we found that Ritonavir, a previously known GLUT inhibitor [11, 12], could dramatically block WST-8 reduction but not intracellular ATP homeostasis. Taken together, our data demonstrate that cellular glucose metabolism is essential for WST-8 reduction and indicate a novel application of tetrazolium-based assays to identify inhibitors or drugs targeting cellular glucose metabolism.

\section{Results}

\section{Medium glucose supply is essential for tetrazolium dye reduction}

In order to investigate the role of medium glucose supply on tetrazolium dye reduction in human cells, we treated immortalized human embryonic kidney 293 cells and cervical carcinoma Hela cells in DMEM with or without D-glucose for $3 \sim 5$ hours and then performed the tetrazolium assays. Four commercially available tetrazolium compounds were used: MTT, MTS, WST- 1 and WST-8/CCK-8 (notably, MTS, WST-1 and CCK-8, but not MTT, were used in combination with PMS.) As shown in Figure 1A 1D, withdrawal of extracellular glucose greatly inhibited tetrazolium dye reduction in both HEK293 and Hela cells. Moreover, WST-8 reduction is glucose concentration dependent (Figure 1E), but not serum dependent (Figure 1F). Notably, it is saturated at the dose of $5 \mathrm{mM}$ of glucose, which is the physiological blood glucose level in humans [13], indicating that the contribution of medium glucose supply to WST-8 reduction depended on the cellular capacity for glucose uptake and utilization.

\section{Tetrazolium dye reduction defect induced by short-term glucose deprivation is not due to cell death}

Glucose deprivation induces cell death [14]. In order to rule out the possibility that the tetrazolium dye reduction defect induced by short-term glucose withdrawal was an outcome of cell death, we took photos for those cells treated as in Figure 1A under microscopy. As shown in Figure 2A, the cell morphology of HEK293 cells treated with glucose deprivation was similar to controls. Furthermore, we incorporated intracellular ATP measurement as an indicator of cellular energy metabolic activity. As shown in Figure 2B, glucose withdrawal greatly attenuated WST-8 reduction (left panel) by not ATP level (right panel), indicating that the WST-8 reduction defect might not be due to cell death. Moreover, in order to show that glucose-starved cells are still capable of reducing tetrazolium when glucose is available, HEK293 and Hela cells were first cultured in glucose free medium for 3 hours, and then re-treated with glucose and tested in the WST-8 assay (Figure 2C). As shown in Figure 2D and 2E, glucose re-treatment could partly re-constitute WST-8 reduction in both HEK293 and Hela cells. Taken together, these data demonstrated that short-term glucose deprivation did not induce cell death.

The role of glucose on WST-8 reduction could not be substituted by glutamine, fructose or galactose

Glucose and glutamine are the two major energy sources for in-vitro cultured cells. Therefore, we investigated whether glutamine could re-constitute the defect of tetrazolium dye reduction induced by glucose deprivation. Cells were treated for 3 hours under glucose and glutamine depleted medium 
supplemented with or without glucose or glutamine. As shown before, WST-8 reduction was greatly attenuated in cells cultured with glucose and glutamine depleted medium, even supplemented with glutamine, but not glucose (Figure 3A). This data demonstrated that glutamine could not re-constitute the WST-8 reduction. Notably, ATP levels in the cells were not changed, even treated with glucose or glutamine depleted medium for 3 hours (Figure 3B), indicating that short-term glucose and glutamine deprivation would not induce cell death in both HEK293 and Hela cells.

In addition to glucose, several other types of monosaccharide play roles in our culture system providing energy and reducing power, such as fructose [15, 16] and galactose [17]. More than 30 years ago, it had been recognized that galactose or fructose could enter glycolytic pathways and support cancer cells to proliferate in the absence of glucose [18]. In order to investigate the specificity of glucose's effect on WST-8 reduction, we tested the effect of other types of monosaccharide, such as D-fructose and
$\mathrm{D}(+)$-galactose on WST-8 reduction. As shown in Figure 3C and 3D, neither galactose nor fructose had a significant effect on WST-8 reduction in both HEK293 and Hela cells when stimulated for 6 hours.

In order to investigate whether HEK293 cells could utilize galactose and fructose, we treated these cells in glucose and glutamine depleted medium supplemented with or without glucose, galactose, fructose or glutamine for 21 hours. As shown in Figure $3 \mathrm{E}$ and $3 \mathrm{~F}$, withdrawal of both glucose and glutamine in HEK293 cells for 21 hours abolished both ATP and WST-8 reduction, while supplementing with glucose and to a much lesser extent, galactose, fructose and glutamine reconstituted cellular ATP and WST-8 reduction, indicating that glutamine, galactose and fructose can to some extent be utilized by the cells.

Therefore, the role of glucose on WST-8 reduction is specific and could not be substituted by glutamine or other monosaccharide, such as fructose or galactose.

\section{A}

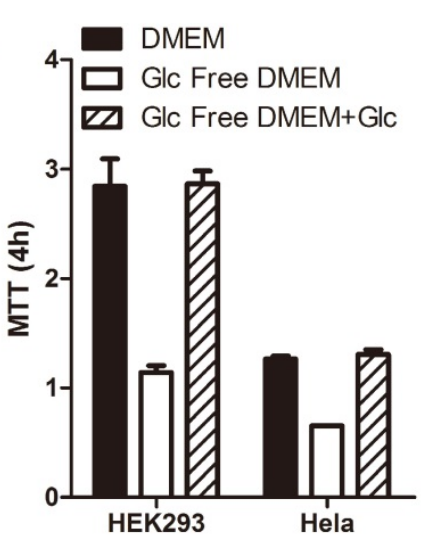

D

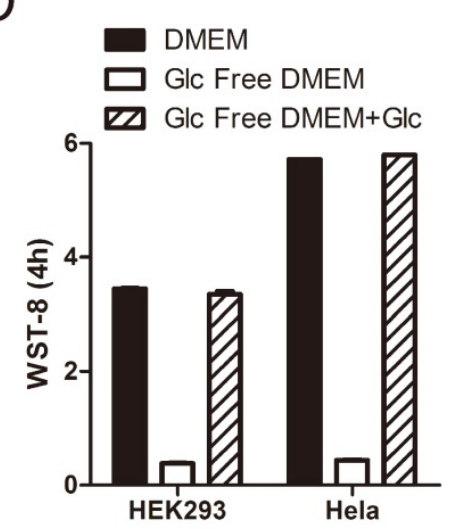

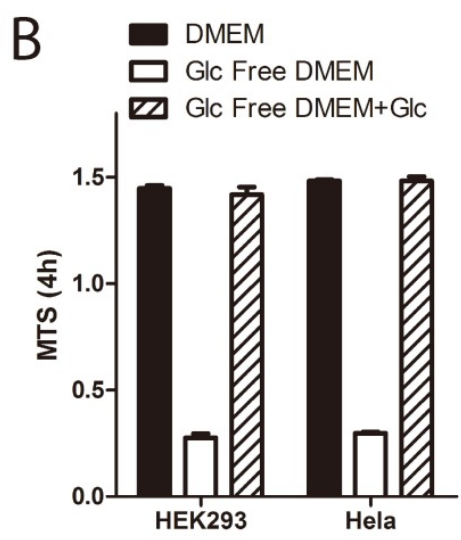

$\mathrm{E}$

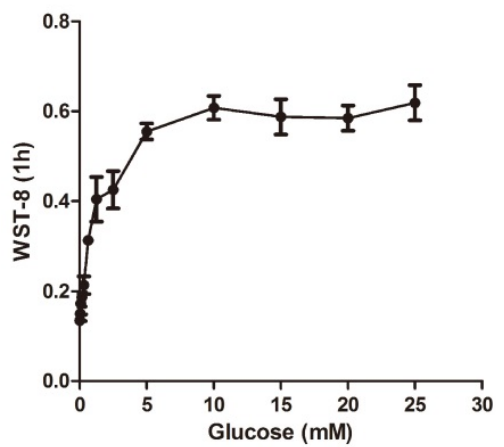

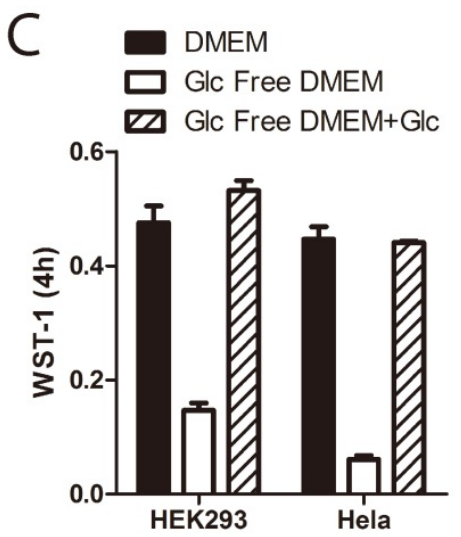

$\mathrm{F} \rightarrow \mathrm{PBS}$

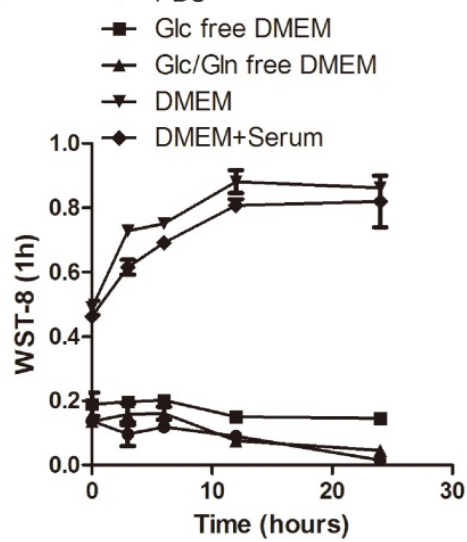

Figure 1. Medium glucose supply is essential for tetrazolium dye reduction. (A D) HEK293 and Hela cells were cultured in the medium as indicated for 3 hours. Tetrazolium-based assays were performed using MTT (A), MTS (B), WST-1 (C) or WST-8 (D). Absorbance was recorded after 4hours incubation. (E) HEK293 cells were cultured in glucose free DMEM supplemented with increasing doses of D-glucose for 3 hours. WST-8 assay was performed. Absorbance was recorded after 1 hour incubation. (F) HEK293 cells were cultured in medium as indicated for 3 hours. WST-8 assay was performed. Absorbance was recorded after 1 hour incubation. Data of three independent replicates are presented as the mean+/-s.e.m., $n=3$. 


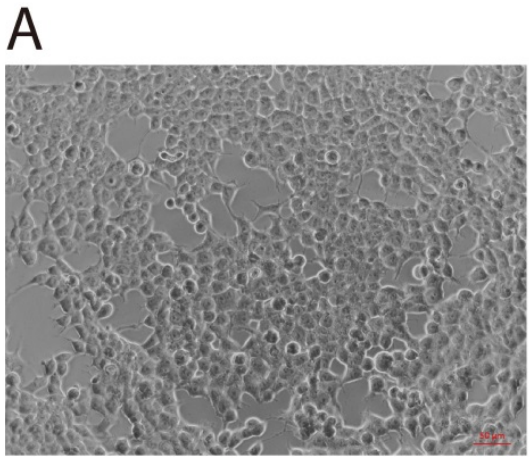

DMEM
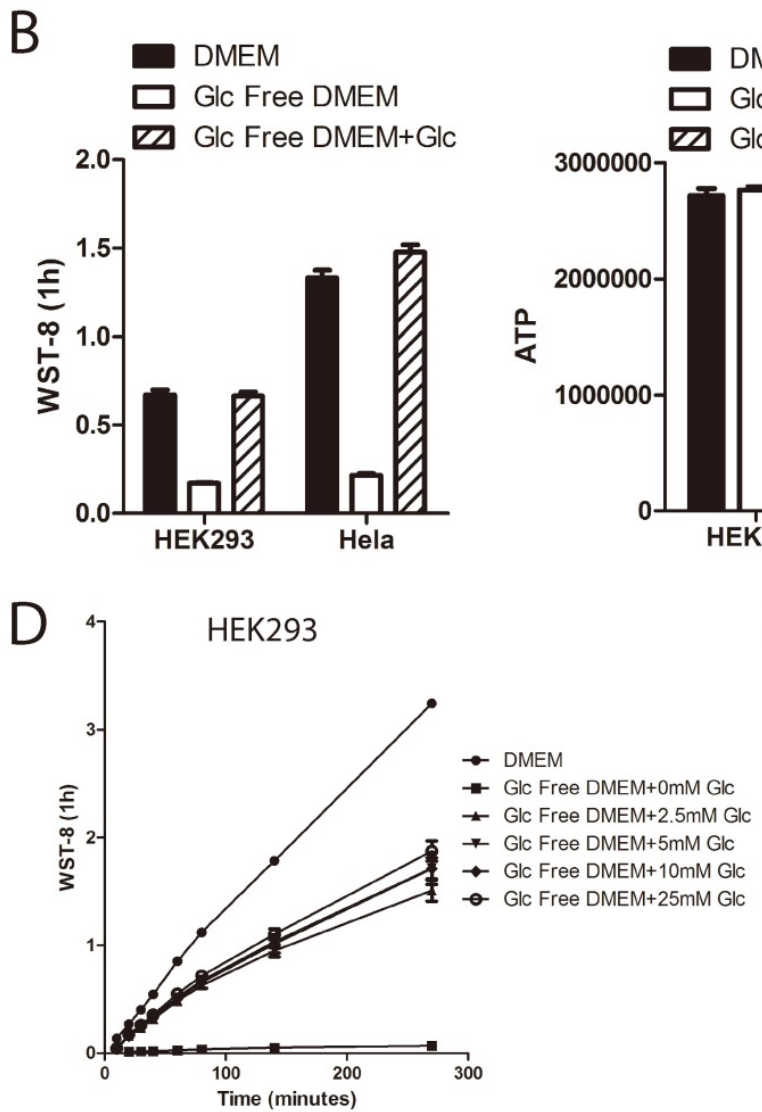

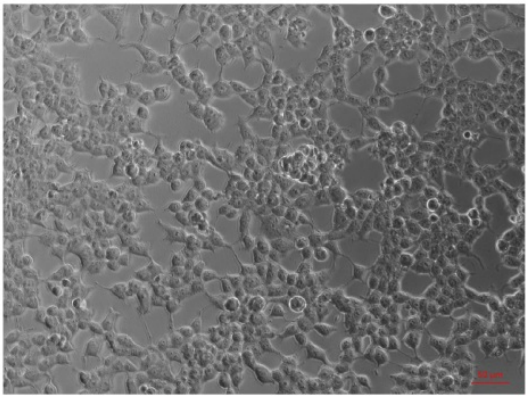

Glc Free DMEM

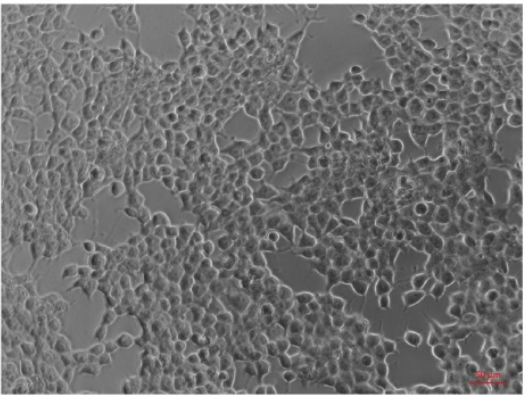

Glc Free DMEM+Glc
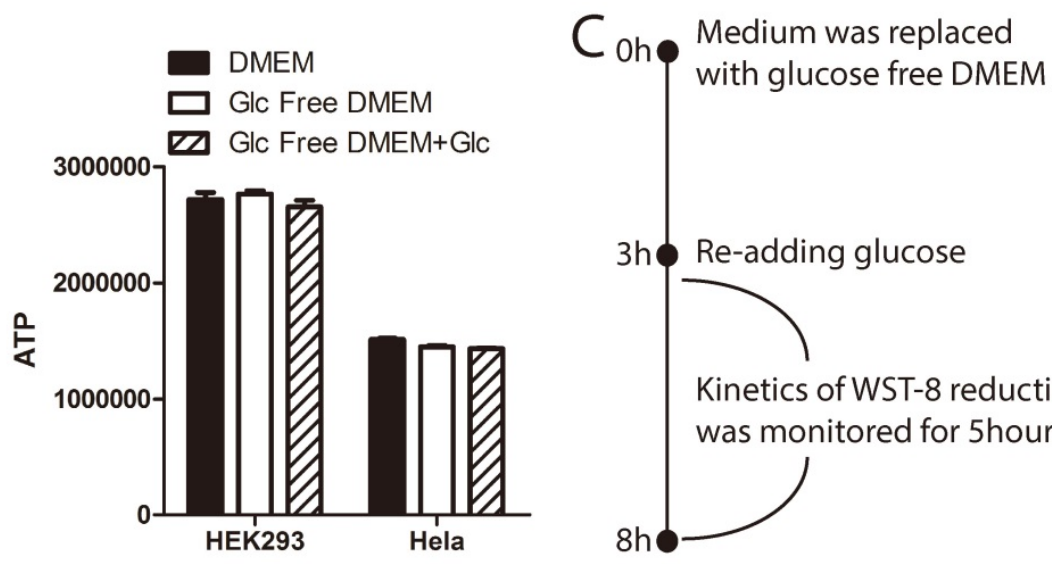

Kinetics of WST-8 reduction was monitored for 5hours

E

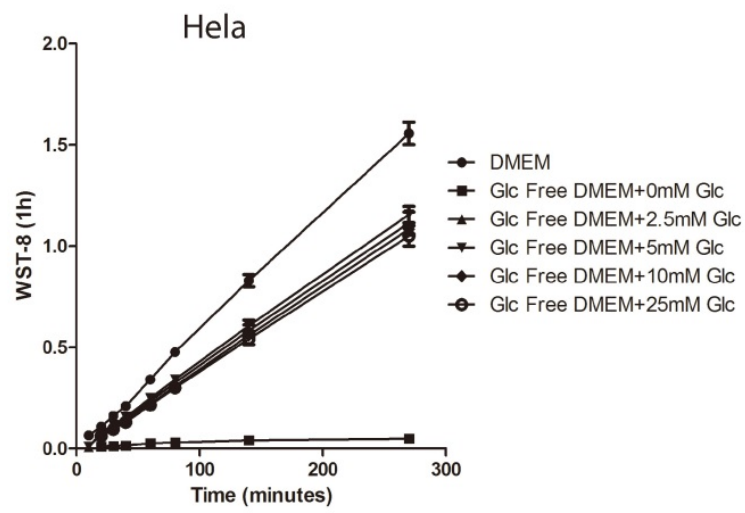

Figure 2. Tetrazoli dye reduction defect induced by short-term glucose deprivation is not due to cell death. (A B) HEK293 cells were cultured in medium as indicated for 3 hours. Cell morphology was photographed under microscopy (A). WST-8 assay was performed and absorbance was recorded after 1 hour incubation (B, left panel). Intracellular ATP level was measured using ATP assay (B, right panel). (C E) HEK293 or Hela cells were cultured in glucose free DMEM for 3 hours. Increasing amount of D-glucose was re-added, and WST-8 assay was performed. Absorbance was then monitored during the next 5 hours. Experiment design is presented in the diagram(C). Absorbance of WST-8 formazan was recorded in HEK293 (D) and Hela cells (E). Data of three independent replicates are presented as the mean+/-s.e.m., $n=3$.

\section{Inhibition of cellular glucose metabolism blocks WST-8 reduction}

Although it has been shown that the source of the WST-8 reduction is trans-plasma membrane electron transport involving $\mathrm{NAD}(\mathrm{P}) \mathrm{H}$-oxidoreductase, other sources of superoxide could also reduce WST-8[19]. In order to elucidate whether inhibition of WST-8 reduction was due to loss of electron transfer with concomitant increase in superoxide generation that was SOD sensitive, we compared the levels of WST-8 reduction in the presence and absence of bovine SOD1. As shown in Supplementary Figure 1, bovine SOD1 indeed partially inhibited WST-8 reduction, indicating superoxide contributed to electron transfer to WST-8. However, the percentage of inhibition was less than $30 \%$ in glucose containing medium, indicating majority of inhibition was due to decreased activity of electron transfer to WST- 8 by a mechanism not involving superoxide. Glucose transport is the first step for cells to utilize glucose. We next asked whether transport of medium glucose across cell membrane is required for tetrazolium dye 
reduction. We used the glucose transporter inhibitor (GLUT-inhibitor II) to inhibit glucose transport through the membrane. As shown in Fig. 4A, GLUT-inhibitor II inhibited WST-8 reduction within 6 hours in a dose dependent manner.

After entering the cells, glucose is first phosphorylated by hexose kinase to produce glucose-6-phosphate before further metabolism. Hexose kinase is also a limiting step for glucose metabolism in the cells. Therefore, we examined whether hexose kinase inhibitor 2-deoxy-glucose (2-DG)[20] could suppress WST-8 reduction. As shown in Fig. 4B, 2-DG strongly suppressed WST-8 reduction in a dose dependent manner. IC50 for 2-DG was around $25 \mathrm{mM}$, which is the same as the glucose concentration in the medium.

Glucose-6-phosphate enters two major metabolism pathways. Through glycolysis, one molecule of glucose-6-phosphate is metabolized into two molecules of pyruvate, producing two molecules of ATP and NADH. Through the pentose phosphate pathway, one molecule of glucose-6-phosphate is metabolized into pentose and NADPH. Both NADH and NADPH are involved in WST-8 reduction. Therefore, we next examined whether iodoacetate targeting GAPDH [21] or DHEA targeting G6PD[22] had any effect on WST-8 reduction. As shown in Fig. $4 \mathrm{C}$ and $4 \mathrm{D}$, both iodoacetate and DHEA attenuated WST-8 reduction in a dose dependent manner.

Inhibition of glucose metabolism leads to increased AMP:ATP ratio, which is sensed by AMPK-mTORC1 pathway. Indeed, treatment with GLUT inhibitor II, 2-DG or DHEA lead to activation of AMPK, indicated by increase of phosphorylation of Acetyl-CoA carboxylase (ACC) on serine-79 and phosphorylation of Raptor on serine-792, and inactivation of mTORC1 indicated by de-phosphorylation of S6K1 on threonine-389 (Figure $4 \mathrm{E})$.

Taken together, these results demonstrate that cellular glucose metabolism is essential for WST-8 reduction.
A

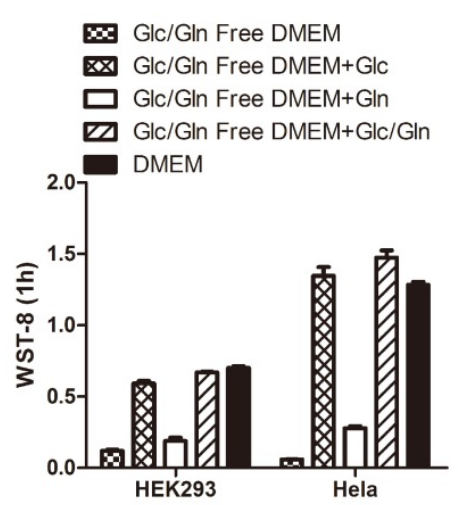

D

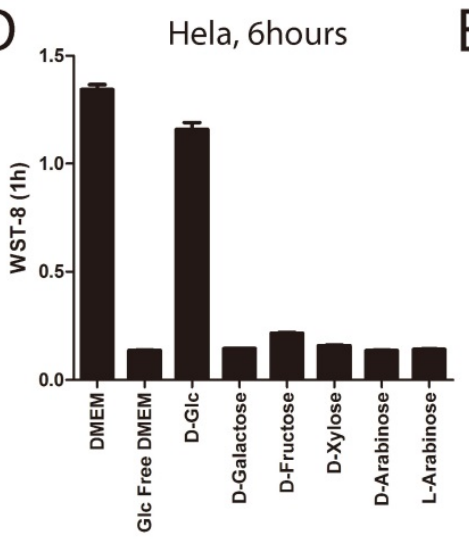

B

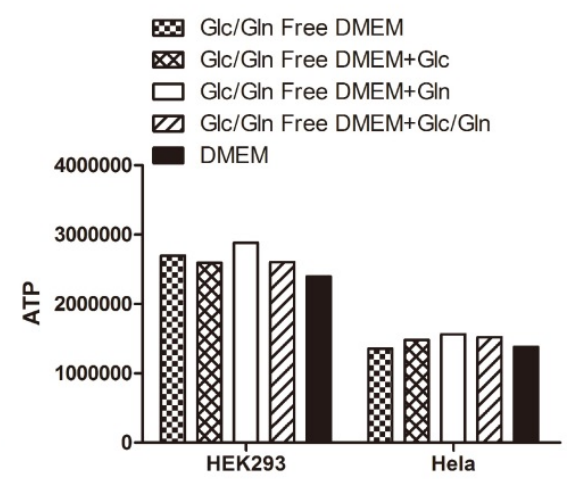

E

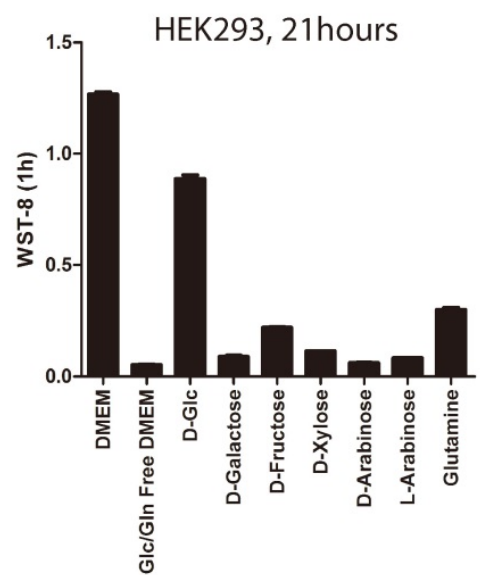

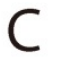

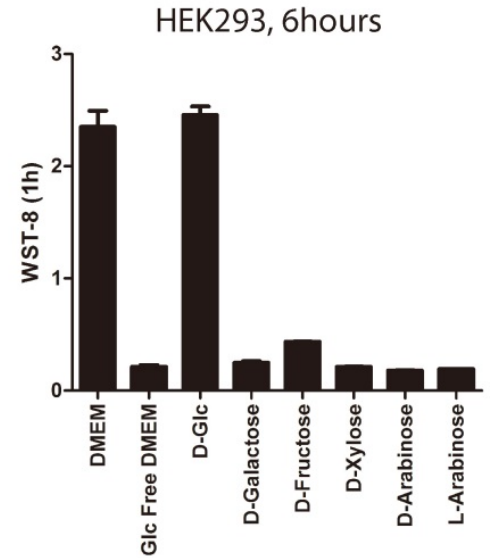

F

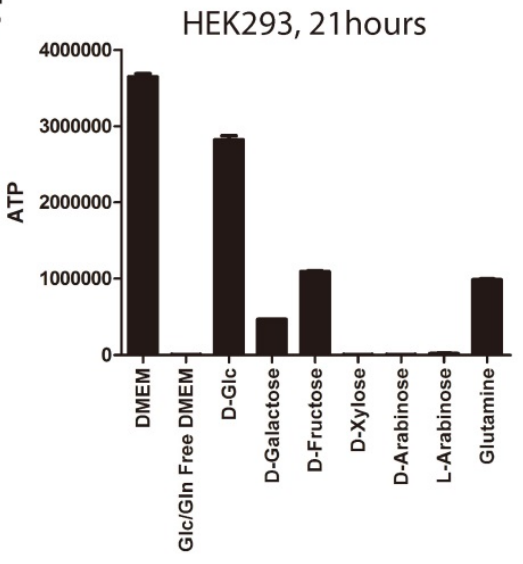

Figure 3. The role of glucose on WST-8 reduction could not be substituted by glutamine, fructose or galactose. (A B) HEK293 and Hela cells were cultured in the medium as indicated for 6 hours. Absorbance of WST-8 formazan was recorded after incubation for 1 hour (A). Intracellular ATP was measured by ATP assay (B). (C D). HEK293 and Hela cells were cultured in DMEM, glucose free DMEM supplemented with or without $25 \mathrm{mM}$ D-glucose, D-galactose, D-fructose, D-xylose, D-arabinose or L-arabinose for 6 hours. Absorbance of WST-8 formazan was recorded in HEK293 (C) and Hela (D) cells. (E F) HEK293 cells were cultured in DMEM, glucose/glutamine free DMEM supplemented with or without $25 \mathrm{mM}$ D-glucose, D-galactose, D-fructose, D-xylose, D-arabinose, L-arabinose or 4mM glutamine for 21 hours. Absorbance of WST-8 formazan was recorded (E). Intracellular ATP level was measured by ATP assay (F). Data of three independent replicates are presented as the mean+/-s.e.m., $\mathrm{n}=3$. 

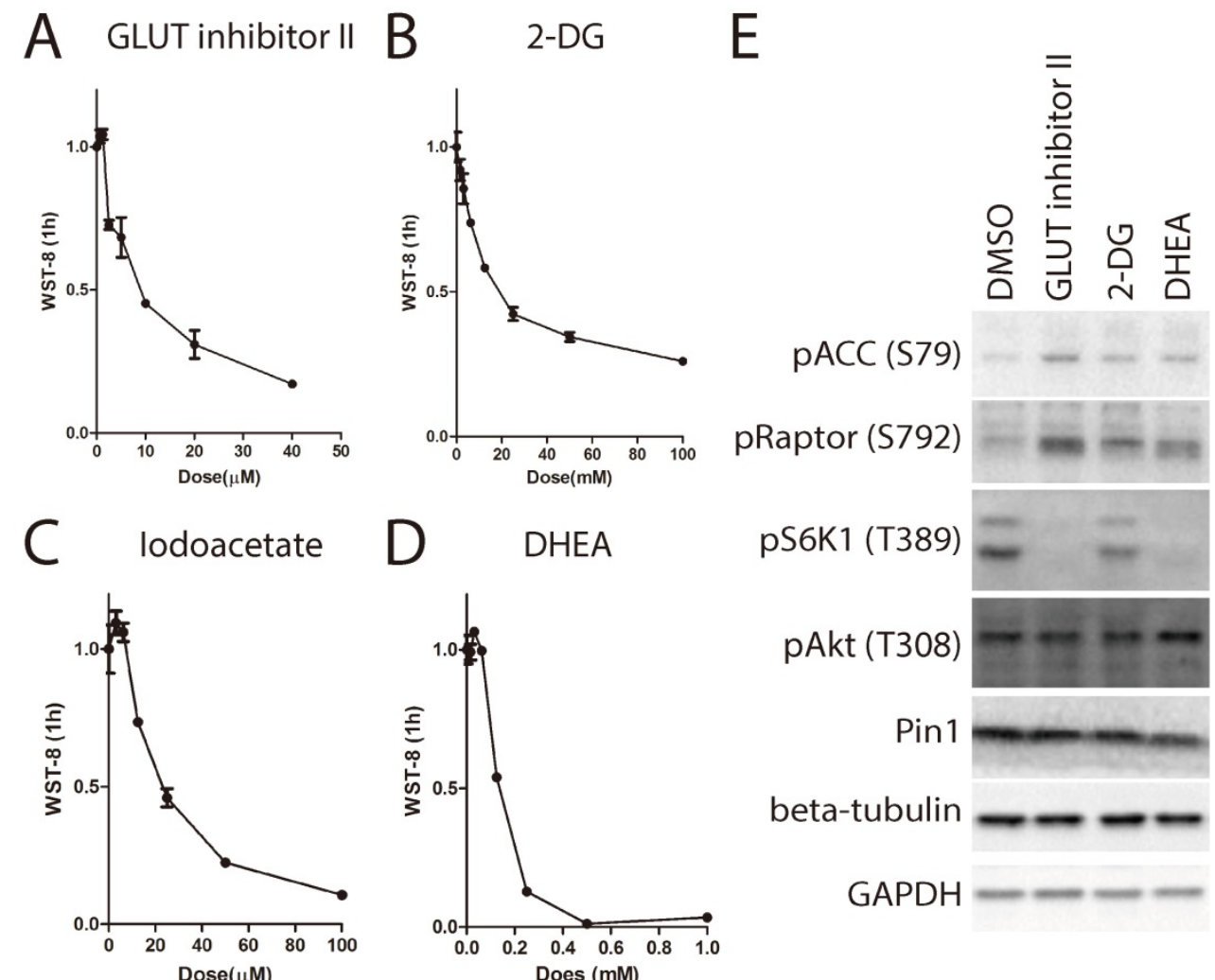

Figure 4. Inhibition of cellular glucose metabolism blocks WST-8 reduction. (A D) Increasing amount of inhibitors was used to treat HEK293 cells for 6 hours. Absorbance of WST-8 formazan was recorded after incubation for 1 hour. Data of GLUT inhibitor II (A), hexokinase inhibitor 2-DG (B), GAPDH inhibitor lodoacetate (C) and G6PD inhibitor (DHEA) (D) were presented. (E) HEK293 cells were treated with inhibitors including GLUT inhibitor II (25 $\mu M)$, 2-DG (25mM), DHEA (62.5 $\mu$ M) for 6 hours. Phosphorylation of ACC on serine-79, Raptor on serine-792, S6K1 on threonine-389, Akt on threonine-308 and expression of protein interacting with NIMA (Pin 1), beta-tubulin or GAPDH were determined by Western Blot.

\section{Inhibition of EGFR-PI3K-Akt pathway attenuates WST-8 reduction}

Due to the well-known role of EGFR/PI3K/Akt pathway in the regulation of glucose metabolism [9, 23], we examined the effect of EGFR signaling pathway inhibitor panel containing 13 inhibitors on WST-8 reduction. As shown in Figure 5A 5C, treatment with EGFR inhibitor, PI3K inhibitor LY294002 and Akt inhibitor VIII for 6 hours greatly inhibited WST-8 reduction in a dose dependent manner. By contrast, inhibitors targeting mTORC1 or MEK had little effect (Figure 5D and 5E). In support of this, the EGFRi, LY294002 and Akt inhibitor VIII, but not rapamycin or PD98059, inhibited the phosphorylation of Akt on Serine-473 (Figure 5F). Interestingly, Akt has been shown to induce GLUT1 expression and membrane localization [24]. Therefore, these data support our notion that WST- 8 reduction is tightly regulated by cellular glucose metabolism.

\section{WST-8 assay identifies ritonavir as an inhibitor of cellular glucose metabolism}

In light of the essential role of cellular glucose metabolism on WST-8 reduction, we next asked whether the WST-8 assay could be used to identify drugs targeting cellular glucose metabolism. We proposed that drugs with inhibitory effects on cellular glucose metabolism might increase the level of blood glucose, leading to hyperglycemia. Several types of drugs are associated with hyperglycemia, such as atypical antipsychotics (clozapine [25]), thiazide and thiazide-Like diuretics (hydrochlorothiazide [26]), corticosteroids (dexamethasone [27]), calcineurin inhibitors (atazanavir) and protease inhibitors (ritonavir [11]). In this study, we investigated whether clozapine, hydrochlorothiazide, dexamethasone or ritonavir had any effect on tetrazolium dye reduction in HEK293 cells. As shown in Figure 6A, ritonavir dramatically inhibited WST-8 reduction in a dose dependent manner. Notably, low dose of ritonavir treatment had a mild effect on intracellular ATP levels (Figure 6B). By contrast, clozapine, dexamethasone or hydrochlorothiazide had only mild effect on WST-8 reduction even at $60 \mu \mathrm{M}$. In support of this, AMPK was activated and mTORC1 was inhibited by ritonavir treatment (Figure 6C). Again, these data not only further support our notion that cellular glucose metabolism is essential for WST-8 reduction, but also unveil a novel application of tetrazolium-based assays in screening for inhibitors or drugs targeting cellular glucose metabolism. 


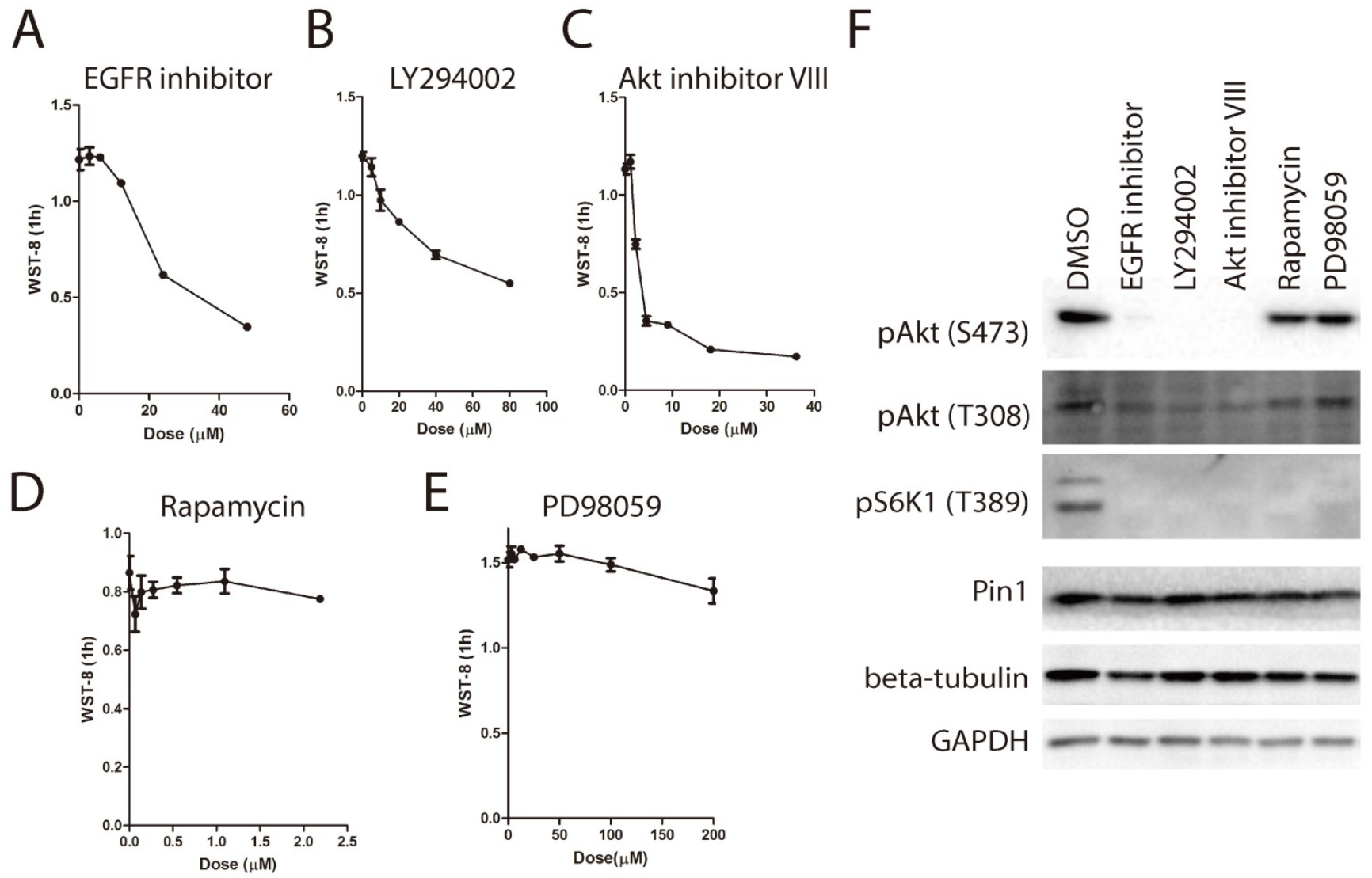

Figure 5. Inhibition of EGFR-PI3K-Akt pathway attenuates WST-8 reduction. (A E) Increasing amount of inhibitors was used treat HEK293 cells for 6 hours. Absorbance of WST-8 formazan was recorded after incubation for 1 hour. Data of EGFR inhibitor (A), PI3K inhibitor LY294002 (B), Akt inhibitor VIII (C), mTORC1 inhibitor Rapamycin (D) and MEK inhibitor PD98059 were presented. Data of three independent replicates are presented as the mean+/-s.e.m., $n=3$. (F) HEK293 cells were treated with inhibitors including EGFR inhibitor ( $3 \mu \mathrm{M})$, LY294002 $(20 \mu \mathrm{M})$, Akt inhibitor VIII $(2 \mu \mathrm{M})$, Rapamycin $(0.5 \mu \mathrm{M})$ and PD98059 (18 $\mu$ M) for 6 hours. Phosphorylation of Akt on serine-473 or threonine-308, phosphorylation of S6K1 on threonine-389, expression of Pinl, tubulin and GAPDH were determined by Western Blot.

A

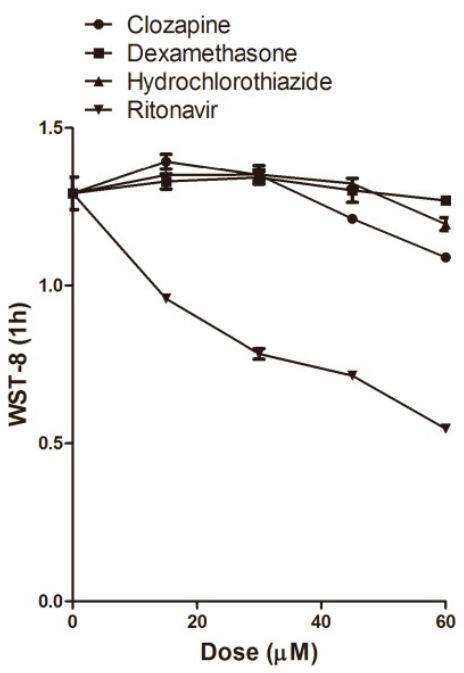

B

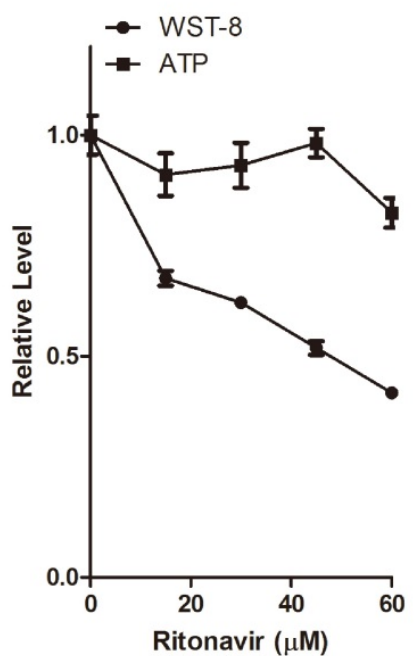

C

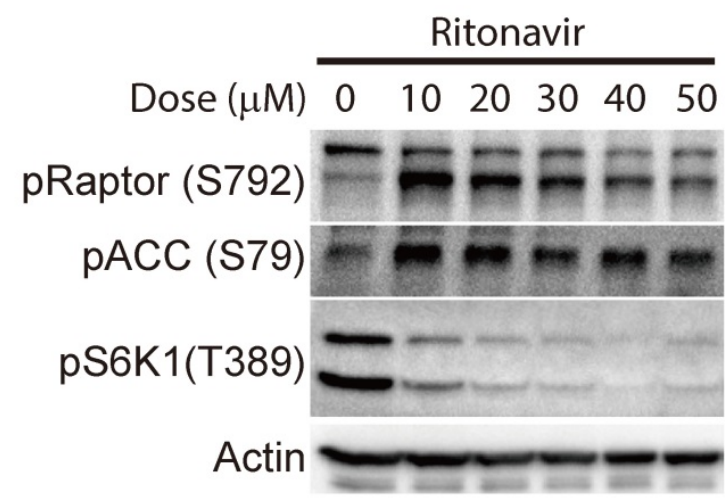

Figure 6. WST-8 assay identifies ritonavir as an inhibitor of cellular glucose metabolism. (A) HEK293 cells were treated with increasing amount of clozapine, dexamethasone, hydrochlorothiazide or ritonavir for 6 hours. Absorbance of WST-8 Formazan was recorded after incubation for 1 hour. (B) HEK293 cells were treated with increasing doses of ritonavir for 6 hours. Absorbance of WST-8 Formazan was recorded after incubation for 1 hour. Intracellular ATP was measured by ATP assay. Relative WST-8 Formazan absorbance or ATP was normalized to its parallel controls. Data of three independent replicates are presented as the mean+/-s.e.m., $n=3$. (C) HEK293 cells were treated with increasing doses of ritonavir. Phosphorylation of ACC on serine-79, Raptor on serine-792, S6K1 on threonine-389, and expression of Actin were determined by Western Blot. 

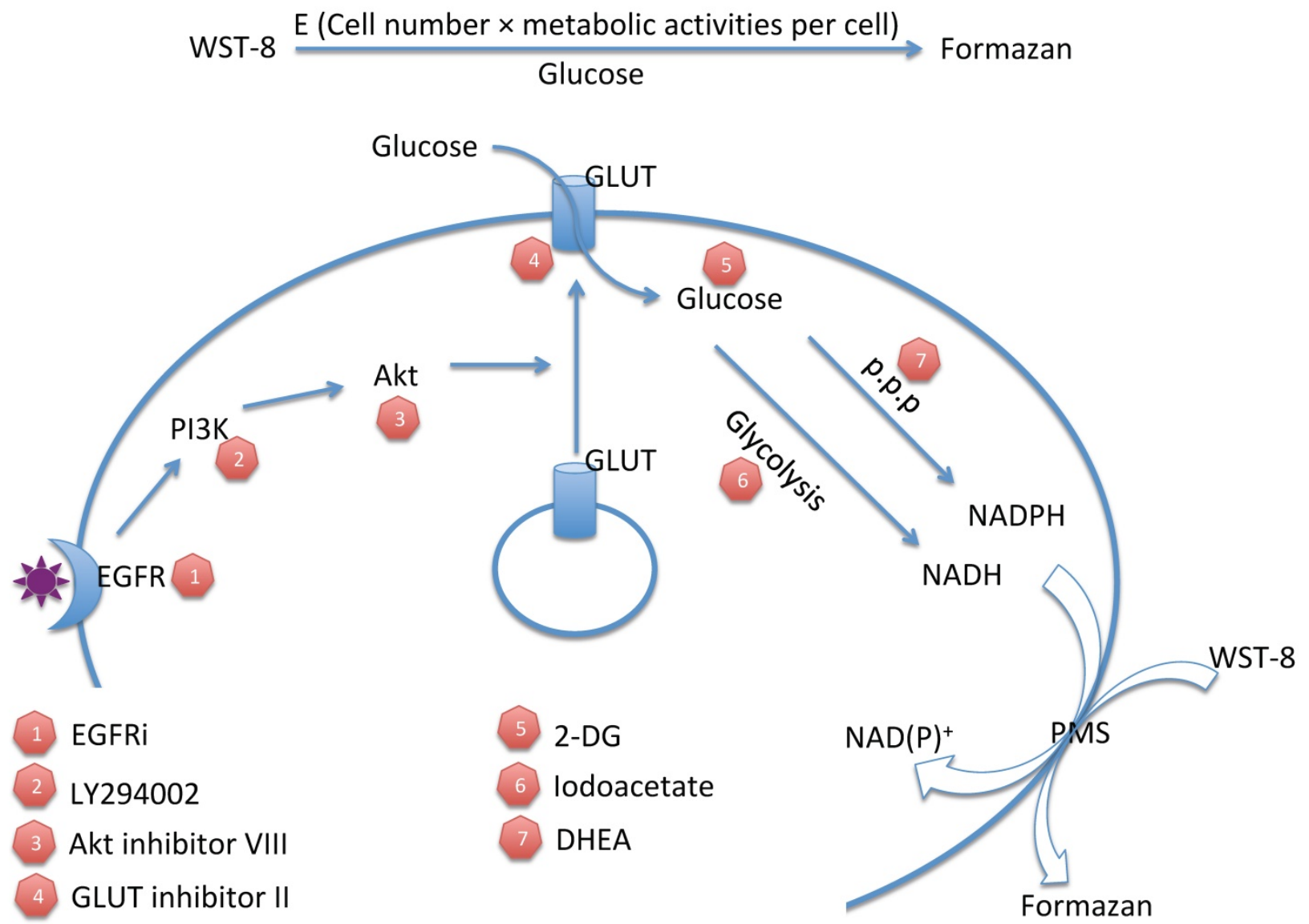

Figure 7. Tetrazolium dye reduction reflects the combinational effect of cell number, medium glucose supply and cellular glucose metabolic activity. WST-8: water-soluble tetrazolium-8. E: total enzyme activities. P.P.P: pentose phosphate pathway. 2-DG: 2-deoxyglucose. EGFRi: EGFR inhibitor. PMS: phenazinium methyl sulfate.

\section{Discussion}

Although tetrazolium-based assays have been widely used for decades, the mechanism of tetrazolium dye reduction remains uncertain. Meanwhile, the conventional application of tetrazolium-based assays in drug screening is performed over several days and based on the assumption that tetrazolium dye reduction is proportional to viable cell number with equal redox metabolic activities[1]. The differences of metabolic activities under different stimulation are usually ignored. However, as implicated previously, tetrazolium dye reduction could be affected by multiple factors, including cell number and growth conditions [2]. Indeed, our study demonstrated that tetrazolium dye reduction reflects the combinational effect of cell number, medium glucose supply and cellular glucose metabolic activity (Figure 7). These results suggest that tetrazolium-based assays are able to indicate not only cell number, but also medium glucose concentration and cellular glucose metabolic activities.

Application of MTT on cellular metabolism could date back to 1990s [28, 29]. In these pioneer works, MTT reduction was compared with
${ }^{3} \mathrm{H}$-thymidine incorporation as early as 30 minutes following use of various metabolic inhibitors. In this study, we performed tetrazolium-based assays followed by treatment of glucose deprivation or glucose metabolic inhibitors within 6 hours, in contrast to several days in the conventional tetrazolium-based assays to monitor cell proliferation. The issue of cell proliferation might be ignored in short times, like 6 hours in mammalian cells. Meanwhile, intracellular ATP measurement was used and indicated cell viability to rule out the possibility that tetrazolium dye reduction defects might be due to cell death. Therefore, combining tetrazolium-based assays with ATP assay could be a novel high-throughput way to monitor cellular glucose metabolism.

Notably, short-term withdrawal of glucose and glutamine has little effect on intracellular ATP levels, but a strong inhibitory effect on tetrazolium dye reduction. However, long-term withdrawal of glucose and glutamine indeed lead to ATP depletion in the cells and probably cell death eventually. These data suggest that tetrazolium-based assays are more sensitive to cellular metabolic blockers than ATP assays. Based on this notion, we proposed that 
tetrazolium-based assays could be a feasible way to screen drugs or inhibitors targeting cellular glucose metabolism. In light of the fact that cancer cells uptake more glucose and have more active glucose metabolism than normal cells [30], tetrazolium-based assays might be helpful to identify cancer types that have more active glucose metabolism and develop anti-cancer drugs targeting cellular glucose metabolism. On the other hand, many normal cells require glucose metabolism for cell survival, so side effects of these drugs might relate to their glucose metabolism-inhibitory properties. In this view, extracellular tetrazolium-based assays might be used as a high-throughput assay to screen those drugs that also target cellular glucose metabolism, avoiding unnecessary side effects. Indeed, in an attempt to identify inhibitors targeting cellular glucose metabolism from hyperglycemia-associated drugs, a previously reported glucose transporter inhibitor and HIV protease inhibitor, named ritonavir, showed dramatic inhibitory effects on tetrazolium dye reduction, but not intracellular ATP levels. Again, it supported the notion that cellular glucose metabolism is essential for tetrazolium dye reduction and that these assays could be used to screen inhibitors or drugs targeting cellular glucose metabolism.

\section{Materials and Methods}

\section{Reagents}

DMEM/High glucose with $4 \mathrm{mM}$ L-Glutamine and $4500 \mathrm{mg} / \mathrm{L}$ glucose (SH30022.01) was purchased from GE Healthcare Life Sciences. Glucose free DMEM with 4mM L-Glutamine (11966-025), Glucose/Glutamine free DMEM without phenol red and sodium pyruvate (A14430-01), Glutamine (A100374) and Fetal bovine serum (10437- 028) were from Thermo Fisher Scientific. D-glucose (A600219), D-galactose (A600215) and D-Fructose (A100226) were from Sangon (Shanghai, China). MTT (M2128), 2-DG (D8375), Iodoacetate (I2512), clozapine (C6305), hydrochlorothiazide (H2910), dexamethasone (D1756), ritonavir (SML0491) and bovine SOD1 (S9697) were from Sigma Aldrich (Shanghai, China). CellTiter 96 Aqueous One Solution Cell Proliferation Assay (G3582) containing MTS and CellTiter-Glo® Luminescent Cell Viability Assay Kit (G7570) were from Promega (Madison, WI, USA). Cell Proliferation Reagent WST-1 (5015944001) was from Roche. Water-Soluble Tetrazolium (WST-8)/Cell counting Kt-8 (CCK-8) (FP101), Beta-tubulin (HC101-02) and GAPDH (HC301-02) were from Transgen (Beijing, China). GLUT inhibitor II (400035), 3-BP (376817), 3-PO (525330), EGFR inhibitor (324674), LY294002 (440202), Akt inhibitor VIII (124018), Rapamycin
(553210) and PD98059 (513000) were from Millipore. DHEA (114331) was from J\&K Scientific Ltd (Beijing, China). Anti-phospho-Raptor (S792) (2083), anti-phospho-ACC (S79) (11818), anti-phospho-S6K1 (T389) (9234), anti-phospho-Akt (S473) (4060), anti-phospho-Akt (T308) (13038) antibodies were from Cell Signaling Technology (Danvers, MA). Anti-Pin1 (Protein interacing with NIMA 1) (10495-1-AP) antibody was from Proteintech (Wuhan, China).

\section{Cell culture and stimulation}

Human embryonic kidney HEK293 cells and human cervical cancer Hela cells were from Cell Bank of Chinese Academy of Sciences. HEK293 cells were cultured in DMEM supplemented with $10 \%$ serum. Hela cells were cultured in MEM supplemented with $10 \%$ serum. For cell stimulation, cells were treated with stimuli diluted in $0.5 \%$ of DMSO (volume to the total volume of the medium).

\section{MTT assay}

$5 \mathrm{mg} / \mathrm{mL}$ MTT (dissolved in PBS) was added in an amount equal to culture volume for each well. Cells were then incubated at $37^{\circ} \mathrm{C}$ with $5 \% \mathrm{CO}_{2}$ for 4hours; culture fluid was removed and disposed. $150 \mu \mathrm{L}$ of MTT solvent $(4 \mathrm{mM} \mathrm{HCl}, 0.1 \%$ Nondet P-40 in isopropanol) was added. Plates were gently stirred in a gyratory shaker to enhance dissolution and read at 560nm using Multiscan GO from Thermo Scientific.

\section{MTS/WST-1/WST-8 assays}

$10 \mu \mathrm{L}$ of MTS/WST-1/WST-8 was directly added into $100 \mu \mathrm{L}$ medium. Cells were then incubated at $37^{\circ} \mathrm{C}$ with $5 \% \mathrm{CO}_{2}$ for 1 hours. Shake thoroughly for 1 minute on a shaker. Absorbance of the samples was measured using Multiscan GO from Thermo Scientific. Wavelength is $490 \mathrm{~nm}$ for MTS formazan, $440 \mathrm{~nm}$ with $630 \mathrm{~nm}$ as reference for WST-1, and $450 \mathrm{~nm}$ with $630 \mathrm{~nm}$ as reference for WST-8.

\section{ATP assay}

Intracellular ATP was measured according to the instruction of CellTiter-Glo ${ }^{\circledR}$ Luminescent Cell Viability Assay Kit (\#G7570, Promega). Briefly, equal volume of CellTiter-Glo® regent was directly added to the culture media. Luminescence was recorded in opaque-walled 96-well plates using Microplate Luminometer (Orion-L2) from Berthord, Germany.

\section{Statistical analysis}

Independent experiments were routinely repeated at least three times. All data are presented as the means \pm s.e.m., followed by determining the significance using the two-tailed student $t$ test. 


\section{Supplementary Material}

Supplementary figure.

http://www.ijbs.com/v14p1535s1.pdf

\section{Acknowledgements}

This research was supported by the Natural Science Fund for Distinguished Young Scientist of Fujian Province (No. 2015J06017), the program of the young university outstanding research talents in Fujian province (No. JA14129), the Joint Science and Technology Innovation Fund Project of Fujian Province (No. 2016Y9043), the Young and Middle-aged Key Personnel Training Program from Fujian Provincial Health and Family Planning Commission (No. 2017-ZQN-58), Fujian environmental protection science and technology project (No. 2016R015), the Natural Science Foundation of Fujian Province (No. 2015J01293), the Collaborative Innovation Center for Stem Cells Translational Medicine (Fujian 2011 Program).

\section{Competing Interests}

The authors have declared that no competing interest exists.

\section{References}

1. Berridge MV, Herst PM, Tan AS. Tetrazolium dyes as tools in cell biology: new insights into their cellular reduction. Biotechnology annual review. 2005; 11: $127-52$.

2. Marshall NJ, Goodwin CJ, Holt SJ. A critical assessment of the use of microculture tetrazolium assays to measure cell growth and function. Growth regulation. 1995; 5: 69-84.

3. Twentyman PR, Luscombe M. A study of some variables in a tetrazolium dye (MTT) based assay for cell growth and chemosensitivity. Br J Cancer. 1987; 56: 279-85.

4. Mattson AM, Jensen CO, Dutcher RA. Triphenyltetrazolium Chloride as a Dye for Vital Tissues. Science. 1947; 106: 294-5.

5. Mosmann T. Rapid colorimetric assay for cellular growth and survival: application to proliferation and cytotoxicity assays. Journal of immunological methods. 1983; 65: 55-63.

6. Alley MC, Scudiero DA, Monks A, Hursey ML, Czerwinski MJ, Fine DL, et al. Feasibility of drug screening with panels of human tumor cell lines using a microculture tetrazolium assay. Cancer research. 1988; 48: 589-601.

7. Vistica DT, Skehan P, Scudiero D, Monks A, Pittman A, Boyd MR. Tetrazolium-based assays for cellular viability: a critical examination of selected parameters affecting formazan production. Cancer research. 1991; 51: 2515-20.

8. Janjic D, Wollheim CB. Islet cell metabolism is reflected by the MTT (tetrazolium) colorimetric assay. Diabetologia. 1992; 35: 482-5.

9. Makinoshima H, Takita M, Saruwatari K, Umemura S, Obata Y, Ishii G, et al. Signaling through the Phosphatidylinositol 3-Kinase (PI3K)/Mammalian Target of Rapamycin (mTOR) Axis Is Responsible for Aerobic Glycolysis mediated by Glucose Transporter in Epidermal Growth Factor Receptor (EGFR)-mutated Lung Adenocarcinoma. The Journal of biological chemistry. 2015; 290: 17495-504

10. Hong SY, Yu FX, Luo Y, Hagen T. Oncogenic activation of the PI3K/Akt pathway promotes cellular glucose uptake by downregulating the expression of thioredoxin-interacting protein. Cellular signalling. 2016; 28: 377-83.

11. Vyas AK, Koster JC, Tzekov A, Hruz PW. Effects of the HIV protease inhibitor ritonavir on GLUT4 knock-out mice. The Journal of biological chemistry. 2010; 285: 36395-400.

12. Hresko RC, Hruz PW. HIV protease inhibitors act as competitive inhibitors of the cytoplasmic glucose binding site of GLUTs with differing affinities for GLUT1 and GLUT4. PloS one. 2011; 6: e25237.

13. Daly ME, Vale C, Walker M, Littlefield A, Alberti KG, Mathers JC. Acute effects on insulin sensitivity and diurnal metabolic profiles of a high-sucrose compared with a high-starch diet. The American journal of clinical nutrition. 1998; 67: 1186-96.
14. Graham NA, Tahmasian M, Kohli B, Komisopoulou E, Zhu M, Vivanco I, et al. Glucose deprivation activates a metabolic and signaling amplification loop leading to cell death. Molecular systems biology. 2012; 8: 589.

15. Park TJ, Reznick J, Peterson BL, Blass G, Omerbasic D, Bennett NC, et al. Fructose-driven glycolysis supports anoxia resistance in the naked mole-rat. Science. 2017; 356: 307-11.

16. Chen WL, Wang YY, Zhao A, Xia L, Xie G, Su M, et al. Enhanced Fructose Utilization Mediated by SLC2A5 Is a Unique Metabolic Feature of Acute Myeloid Leukemia with Therapeutic Potential. Cancer cell. 2016; 30: 779-91.

17. Coelho AI, Berry GT, Rubio-Gozalbo ME. Galactose metabolism and health. Curr Opin Clin Nutr Metab Care. 2015; 18: 422-7.

18. Hamanaka RB, Chandel NS. Targeting glucose metabolism for cancer therapy. The Journal of experimental medicine. 2012; 209: 211-5.

19. Peskin AV, Winterbourn CC. Assay of superoxide dismutase activity in a plate assay using WST-1. Free radical biology \& medicine. 2017; 103: 188-91.

20. Penny HL, Sieow JL, Adriani G, Yeap WH, See Chi Ee P, San Luis B, et al. Warburg metabolism in tumor-conditioned macrophages promotes metastasis in human pancreatic ductal adenocarcinoma. Oncoimmunology. 2016; 5; e1191731.

21. Devalaraja-Narashimha K, Padanilam BJ. PARP-1 inhibits glycolysis in ischemic kidneys. Journal of the American Society of Nephrology : JASN. 2009; 20: 95-103.

22. Zhang Z, Chen $X$, Jiang $C$, Fang $Z$, Feng $Y$, Jiang $W$. The effect and mechanism of inhibiting glucose-6-phosphate dehydrogenase activity on the proliferation of Plasmodium falciparum. Biochimica et biophysica acta. 2017; 1864: 771-81.

23. Makinoshima H, Takita M, Matsumoto S, Yagishita A, Owada S, Esumi H, et al. Epidermal growth factor receptor (EGFR) signaling regulates global metabolic pathways in EGFR-mutated lung adenocarcinoma. The Journal of biological chemistry. 2014; 289: 20813-23

24. Zhou QL, Jiang ZY, Holik J, Chawla A, Hagan GN, Leszyk J, et al. Akt substrate TBC1D1 regulates GLUT1 expression through the mTOR pathway in 3T3-L1 adipocytes. The Biochemical journal. 2008; 411: 647-55.

25. Lindenmayer JP, Nathan AM, Smith RC. Hyperglycemia associated with the use of atypical antipsychotics. The Journal of clinical psychiatry. 2001; 62 Suppl 23: 30-8.

26. Halprin H. Hyperglycemic reaction to a hydrochlorothiazide. The Journal of the Medical Society of New Jersey. 1960; 57: 254-5.

27. de Oliveira $C$, de Mattos AB, Biz C, Oyama LM, Ribeiro EB, do Nascimento CM. High-fat diet and glucocorticoid treatment cause hyperglycemia associated with adiponectin receptor alterations. Lipids in health and disease. 2011; 10: 11.

28. Berridge MV, Horsfield JA, Tan AS. Evidence that cell survival is controlled by interleukin-3 independently of cell proliferation. Journal of cellular physiology. 1995; 163: 466-76.

29. Berridge MV, Tan AS. Interleukin-3 facilitates glucose transport in a myeloid cell line by regulating the affinity of the glucose transporter for glucose: involvement of protein phosphorylation in transporter activation. The Biochemical journal. 1995; 305 ( Pt 3): 843-51.

30. Warburg O. On the origin of cancer cells. Science. 1956; 123: 309-14. 